DOI: https://doi.org/10.11144/Javeriana.upsy16-3.imri

\title{
Inteligencias múltiples y su relación con inteligencias cognitiva y emocional en adolescentes
}

\section{Multiple Intelligences and their Relationships with Cognitive and Emotional Intelligences in Adolescents}

Recepción: 13 Diciembre 2016 | Aprobación: 27 Enero 2017

\author{
Javier Cejudo a \\ Universidad de Castilla La Mancha, España \\ ORCID: http://orcid.org/0000-0002-2381-0714 \\ Lidia LosAdA \\ Universidad Nacional de Educación a Distancia, \\ España \\ Juan Carlos Pérez-González \\ Universidad Nacional de Educación a Distancia, España
}

\footnotetext{
${ }^{a}$ Autor de correspondencia. Correo electrónico: manueljavier.cejudo@uclm.es

Para citar este artículo: Cejudo, J., Losada, L., \& Pérez González, J. C. (2017). Inteligencias múltiples y su relación con inteligencias cognitiva y emocional en adolescentes. Universitas Psychologica, 16(3), 1-13.

https://doi.org/10.11144/Javeriana.upsy16-3.imri
}

\section{RESUMEN}

A partir del Inventario de Autoeficacia para Inteligencias Múltiples (IAMI), desarrollado en Argentina por Pérez, Beltramino y Cupani (2003), presentamos una adaptación abreviada para adolescentes españoles (IAMI-M40). IAMI evalúa la autoeficacia que los adolescentes tienen sobre las distintas inteligencias múltiples (IIMM) propuestas por Gardner (1999). En una muestra de estudiantes españoles de educación secundaria de 11 a 19 años $(n=313)$, se evaluó su inteligencia fluida (PMA-R), cristalizada (16-PF-R) y emocional (TEIQue-ASF), y su nivel de IIMM mediante una adaptación española. El análisis factorial exploratorio de la adaptación española identificó ocho factores correspondientes a cada tipo de inteligencia del modelo de Gardner (1999), reteniendo 40 ítems de los 69 originales. Los resultados avalan la fiabilidad y la validez convergente del IAMI-M40.

Palabras clave

inteligencias múltiples; autoeficacia; validación; autoinforme; adolescentes

\begin{abstract}
From the Self-Efficacy Inventory for Multiple Intelligences (IAMI), developed in Argentina by Pérez, Beltramino and Cupani (2003), we present a shortened adaptation of this self-report for Spanish adolescents (IAMI-M40). IAMI evaluates the self-efficacy that adolescents have about the different multiple intelligences (IIMM) proposed by Gardner (1999). In a sample of Spanish secondary school students 11 to 19 years ( $n=313)$, its fluid (PMA-R), crystallized (16-PF-R), and emotional intelligence (TEIQue-ASF) and their level of IIMM by an Spanish adaptation of IAMI was assessed. Exploratory factor analysis of the Spanish adaptation of IAMI identified eight factors for each type of intelligence according to the Gardner's (1999) model, holding 40 items of the 69 original. The results support the reliability and convergent validity of IAMI-M40.
\end{abstract}


Keywords

multiple intelligences; self-efficacy; validation; self-report; adolescents

\section{Introducción}

De acuerdo con Klein $(1997,1998)$ una versión débil o suave de la teoría de las inteligencias múltiples (IIMM) carece de interés, mientras que una versión dura o seria, como es la defendida por Gardner (1983, 1998, 1999), está inadecuadamente apoyada por la evidencia que presenta Gardner, además por la prueba en contra acumulada desde otras fuentes científicas. En gran medida la carencia de suficiente apoyo empírico a la teoría de las IIMM se debe a la práctica ausencia de instrumentos fiables y válidos para su evaluación, lo que genera insatisfacción entre profesionales e investigadores de la psicología y de la educación interesados en esta teoría (p. ej., Brody, 1992; Reeve \& Bonaccio, 2011; Roberts \& Lipnevich, 2011; Visser, Ashton, \& Vernon, 2006; Waterhouse, 2006). En su revisión sobre la teoría de las IIMM, Pérez y Beltrán (2006) destacaron la acusación a Gardner de la inexistencia de una batería de instrumentos para identificar y evaluar las diferentes inteligencias de su teoría. Así, en los últimos años, distintos autores (p. ej., Almeida et al., 2010; Roberts \& Lipnevich, 2011) han reivindicado la necesidad de investigación sobre la evaluación de las IIMM.

Hasta la fecha, apenas se dispone de instrumentos de rendimiento máximo (Cronbach, 1949) para la evaluación de las IIMM, con la excepción de la batería de Visser et al. (2006), ejemplo de "test de aptitud", y las Spectrum Activities o SA de Gardner (1998), ejemplo de "test de logro o de competencia", según terminología de Cronbach (1949). Por el contrario, sí se pueden encontrar en la literatura varias escalas de rendimiento típico (autoinformes) dirigidas a evaluar las IIMM: Teele Inventory of Multiple Intelligences o TIMI (Teele, 1992; McMahon, Rose, \& Parks, 2004), Multiple Intelligences Inventory o MII (Armstrong, 1999), Multiple Intelligences Developmental
Assessment Scale o MIDAS (Shearer, 1999, 2004), Student Multiple Intelligences Profile o SMIP (Chan, 2001), Multiple Intelligences Test o MIT (Haselbauer, 2005), Self-Assessed Multiple Intelligence o SAMI (Furnham, 2009). Todos estos autoinformes han sido desarrollados en el ámbito anglosajón, aunque se dispone de adaptación al español del MII (Almeida et al., 2010; Hernández-Torrano, Ferrándiz, Ferrando, Prieto, \& Fernández, 2014; Llor et al., 2012) y del MIDAS (Pizarro \& Clark, 2000; Pizarro et al., 2002).

El Inventario de Autoeficacia para Inteligencias Múltiples o IAMI (Pérez et al., 2003) es el único autoinforme de IIMM desarrollado en un contexto hispanohablante y con una clara vocación de aplicación educativa. Posteriormente, los autores presentaron una revisión del IAMI ([IAMI-R], Pérez \& Cupani, 2008); si bien se trata de una modificación menor, consistente en una reformulación de 12 ítems por problemas de comprensión detectados en una muestra de adolescentes argentinos.

Aunque los autoinformes no constituyen la manera ideal de evaluar las IIMM según Gardner (1998, 2006), sí representan una vía práctica para aproximarse a la evaluación esquiva de las mismas (Armstrong, 1999). Para Petrides (2011), las IIMM no son realmente "inteligencias" en sentido estricto ("capacidades mentales"), sino más bien aspectos o rasgos de la personalidad. Bajo este novedoso prisma ofrecido por Petrides (2011), dado que la evaluación de los rasgos de personalidad es apropiada a través de la utilización de instrumentos de rendimiento típico (autoinformes), sería entonces perfectamente viable utilizar un autoinforme para evaluar las IIMM, si estas se entienden más como rasgos de personalidad que como inteligencias o competencias. En cualquier caso, el IAMI no pretende evaluar "inteligencias", "talentos" o "competencias", sino más bien "percepciones de autoeficacia" (o autoeficacia percibida), es decir, el grado de confianza en la propia capacidad para ser eficaz en (realizar exitosamente) actividades relacionadas con las ocho inteligencias propuestas por Gardner (1999). Así, dado que las percepciones son 
subjetivas, un autoinforme constituye igualmente en este caso un vehículo idóneo de evaluación psicológica (Bandura, 2006).

El objetivo del presente trabajo de investigación es la adaptación y validación de una versión abreviada del IAMI para adolescentes españoles, y se enmarca en la categoría de "estudios instrumentales" (Montero \& León, 2007).

\section{Método}

\section{Muestra}

Para este estudio se empleó un muestreo no probabilístico de tipo incidental o por accesibilidad. La muestra total de participantes estuvo compuesta por 313 alumnos de Educación Secundaria (48.9 \% chicos) con edades comprendidas entre los 11 y los 19 años $(\mathrm{M}=13.37, \mathrm{DE}=1.76)$. Los alumnos participantes pertenecen a dos Institutos de Enseñanza Secundaria de titularidad pública de la Comunidad Autónoma de Castilla La Mancha, España. La distribución de la muestra por cursos de ESO fue: $40.6 \%$ de primer curso, $14.1 \%$ de segundo, $15.3 \%$ de tercero y $9.6 \%$ de cuarto. Por último, los estudiantes de primero de bachillerato constituían el $20.4 \%$.

\section{Instrumentos}

Se utilizó el Inventario de Autoeficacia para Inteligencias Múltiples (IAMI) (Pérez et al., 2003) en su adaptación española realizada para este estudio (en adelante, IAMI-M). Esta prueba está compuesta por ocho subescalas, cada una representando una de las inteligencias propuestas por Gardner (1999). Consta de 69 ítems que incluyen actividades académicas ("Analizar obras literarias"). Se solicita al examinado que evalúe el nivel de confianza para desempeñar exitosamente cada una de las actividades en una escala Likert de 10 puntos: no puedo desempeñar esta actividad (1); estoy completamente seguro de poder desempeñar esta actividad exitosamente (10).
Para la evaluación de la inteligencia general se utilizaron dos indicadores de capacidad cognitiva:

Aptitudes Mentales Primarias-Factor $R$ "Razonamiento" ([PMA], Thurstone \& Thurstone, 1941)

La subescala de razonamiento del PMA evalúa la capacidad para resolver problemas lógicos; consta de 30 ítems y tiene una duración de 6 minutos, siendo un buen indicador de razonamiento inductivo (Hertzog \& Bleckley, 2001; Sánchez-Ruiz, HernándezTorrano, Pérez-González, Batey, \& Petrides, 2011). Se considera un factor de primer orden (i. e., Estrato 1) en la estructura jerárquica de las capacidades cognitivas, estrechamente vinculado a la inteligencia fluida (Gf) (Carroll, 1993). Así, se utilizó el PMA-R como una aproximación de la capacidad cognitiva general, en línea con estudios previos (Román et al., 2014; SánchezRuiz, Hernández-Torrano, Pérez-González, Batey, \& Petrides, 2011). En el presente estudio, la consistencia interna de la subescala fue de 0.87.

Cuestionario 16PF Quinta Edición (Cattell, Cattell, \& Cattell, 1993)

Tiene como objetivo la apreciación de 16 rasgos de primer orden y cinco dimensiones globales de la personalidad. En esta investigación se utilizó únicamente la subescala B: Razonamiento (16PF-r), que consta de 15 ítems $\mathrm{y}$ ofrece una breve medida de inteligencia. Altas puntuaciones indican una satisfactoria capacidad de razonamiento y capacidad verbal. Se utilizó el 16 PF-R como una aproximación de la Gc, en línea con estudios previos ( $\mathrm{O}^{\prime}$ Connor \& Little, 2003). En este estudio, la consistencia interna de la subescala fue de 0.61.

Trait Emotional Intelligence Adolescent Short Form ([TEIQue-ASF], Petrides, Sangareau, Furnham, \& Frederickson, 2006)

Para la evaluación de la Inteligencia Emocional (IE) rasgo (Pérez-González \& Sánchez-Ruiz, 2014) se utilizó la adaptación española (Ferrando et al., 2011) que contiene 30 ítems formulados en una escala Likert de 7 puntos diseñados para medir la IE rasgo global. La consistencia interna para la puntuación IE rasgo global en la muestra fue igual a 0.8 . 


\section{Procedimiento}

La adaptación del IAMI al contexto español (IAMI-M) partió del instrumento original (Pérez et al., 2003), y siguió los estándares metodológicos internacionales recomendados por la International Test Comision (ITC) para la adaptación de instrumentos (p. ej., Muñiz, Elosua, \& Hambleton, 2013).

Tras una primera adaptación gramatical y semántica al contexto español, se analizaron cada uno de los ítems originales del instrumento y se consensuó entre los autores el contenido final del instrumento, realizándose cambios mayores en la redacción de los ítems: 25, 39, 41, 45, 63 y 68. Al estar en la versión original del IAMI los ítems agrupados por dimensión, con el fin de minimizar el posible sesgo que esta agrupación pudiera inducir en el patrón de respuestas, se cambió el orden original de los ítems, quedando entremezclados por pares según la dimensión que evaluaban.

Se obtuvo el consentimiento de los padres y la autorización de los directores de los centros educativos. La administración de los instrumentos se realizó siguiendo las instrucciones de los manuales, en presencia del tutor y en horario lectivo. Se aseguró el carácter anónimo de las respuestas.

\section{Análisis de datos}

Con el fin de reducir la extensión del instrumento y seleccionar los ítems más representativos, se realizó un Análisis Factorial Exploratorio (AFE). Presuponiendo cierta relación entre los factores resultantes, se optó por una rotación oblicua, según la recomendación de Kline (1994), empleando el método de rotación Promax y el valor $k=3$, de acuerdo con las indicaciones de Hendrickson y White (1964).

Se estudió la fiabilidad (consistencia interna) de cada subescala y factor a través del cálculo del alfa de Cronbach y se analizaron las correlaciones entre las puntuaciones correspondientes a cada una de las subescalas. Para analizar las evidencias de validez convergente se estudió la relación de cada subescala con indicadores de inteligencia general (Gf y Gc) e IE rasgo.

\section{Resultados}

\section{Análisis factorial exploratorio}

Previo al AFE, se estudió el índice de adecuación de Kaiser-Meyer-Olkin (KMO), obteniendo un valor de 0.88. El test de esfericidad de Bartlett fue significativo al nivel de $\mathrm{p} \leq 0.001(\chi 2=14746.4$; $\mathrm{df}=2346$ ), mostrando que la intercorrelación entre los ítems fue apropiada para el empleo del análisis factorial.

La regla Kaiser-Guttman, de autovalores superiores a 1 , identificó 14 factores que explicaron un $68.81 \%$ de la varianza de respuesta al inventario. La interpretación del Scree Test de Cattell (1966) para la magnitud de los autovalores, un procedimiento más confiable que el anterior (Kline, 1994; Beavers et al., 2013), indicó la conveniencia de interpretar solo ocho factores, por lo que se volvió a realizar el AFE limitando la extracción a ocho factores rotados.

En la Tabla 1 se muestra la estructura factorial resultante de la escala, suprimiendo las cargas factoriales más reducidas $(<0.3)$. La solución factorial quedó constituida por ocho factores que explicaron un $52.7 \%$ de la varianza.

Factor 1: compuesto de ocho ítems con saturaciones que oscilaron entre 0.91 y 0.49 que explicaron un $23.87 \%$ de la varianza. Todos estos items forman parte de la subescala inteligencia musical del instrumento original.

Factor 2: nueve ítems explicaron un 9.06 $\%$ de la varianza con saturaciones entre 0.85 y 0.37. Todos ellos forman parte de la subescala inteligencia intrapersonal del instrumento original a excepción del ítem 65 ("Iniciar y mantener relaciones con otras personas").

Factor 3: nueve ítems que saturaron con cargas entre 0.85 y 0.63 , y que explicaron un $6.75 \%$ de la varianza. Todos forman parte de la subescala inteligencia cinestésico-corporal del instrumento original. 
Factor 4: nueve ítems que saturaron con cargas entre 0.89 y 0.43 , y que explicaron un $5.62 \%$ de la varianza. Todos forman parte de la subescala inteligencia lógico-matemática del instrumento original a excepción del ítem 61 ("Realizar análisis químicos de compuestos orgánicos”).

Factor 5: ocho ítems que saturaron con cargas entre 0.8 y 0.56 , y que explicaron un $4.42 \%$ de la varianza. Todos forman parte de la subescala inteligencia espacial-visual del instrumento original.

Factor 6: nueve ítems que saturaron con cargas entre 0.81 y 0.34 , y que explicaron un $3.19 \%$ de la varianza. Todos forman parte de la subescala inteligencia lingüística del instrumento original a excepción del ítem 19 ("Comprender estadísticas de encuestas y censos").

Factor 7: ocho ítems que saturaron con cargas entre 0.73 y 0.41 , y que explicaron un $2.93 \%$ de la varianza. Todos forman parte de la subescala inteligencia naturalista del original.

Factor 8: compuesto por nueve ítems que saturaron con cargas entre 0.58 y 0.39 , y que explicaron un $2.34 \%$ de la varianza. Todos forman parte de la subescala inteligencia interpersonal del instrumento original a excepción del ítem 24 (Cantar armoniosamente junto a otras personas).

\section{TABLA 1}

Estructura factorial rotada del IAMI-M

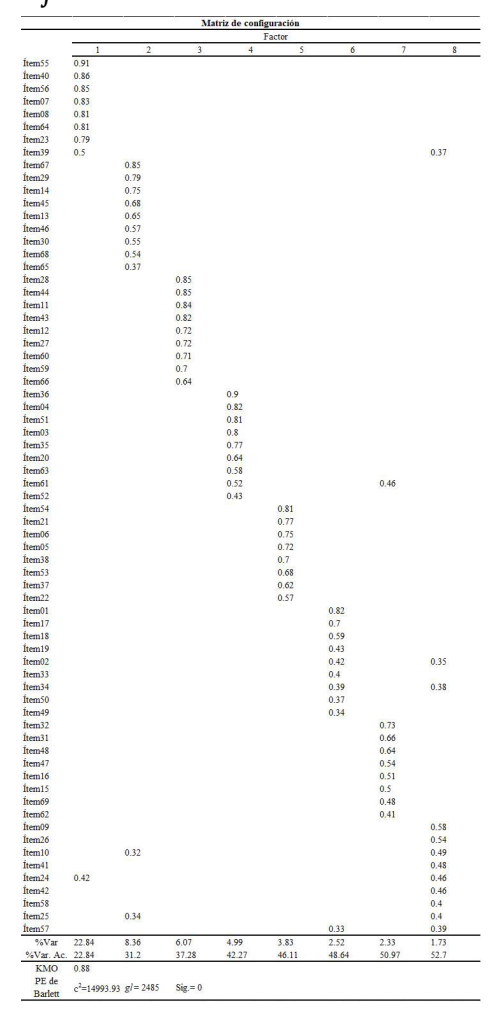

Nota. Estos resultados son fruto de un AFE realizado según el método de extracción de ejes principales y el método de rotación. Promax con $\mathrm{K}=3$ : Las cargas factoriales inferiores a 0.3 han sido omitidas: Factor I: Inteligencia musical; Factor II: Inteligencia intrapersonal; Factor III: Inteligencia cinestésico-corporal; Factor IV: Inteligencia lógico-matemática; Factor V: Inteligencia espacial-visual; Factor VI: Inteligencia lingüística; Factor VII: Inteligencia naturalista; Factor VIII: Inteligencia interpersonal. Fuente: elaboración propia.

\section{Evidencias de fiabilidad}

Definidos los ocho factores, se realizaron análisis descriptivos para cada uno de los ítems del IAMI$\mathrm{M}$, y se hallaron los coeficientes de correlación corregidos de cada ítem con el total de su factor, así como su contribución a la consistencia interna de la subescala.

El intervalo de puntuaciones medias para cada uno de los ítems oscila entre los siguientes valores: 4.12 y 7.78 , siendo en general la subescala inteligencia intrapersonal en la que se obtuvieron puntuaciones más elevadas, y en la 
subescala inteligencia musical, las puntuaciones más bajas. El estudio de la desviación estándar de las puntuaciones de los ítems evidencia una distribución normal de las respuestas de los sujetos, con valores que oscilan entre 1.86 y 3.06. Para el análisis de las correlaciones ítem-total se estableció como criterio mínimo de validez interna el coeficiente 0.2 ; todos los ítems alcanzaron un valor superior. Las correlaciones ítem-total oscilaron entre 0.44 y 0.84. Las medias de las correlaciones ítem-total de cada una de las subescalas fueron las siguientes: subescala inteligencia intrapersonal 0.62; subescala inteligencia musical 0.77 ; subescala inteligencia cinestésica-corporal 0.72; subescala inteligencia lógico-matemática 0.66 ; subescala inteligencia visual-espacial 0.67 ; subescala inteligencia lingüística 0.57 ; subescala inteligencia naturalista $0.54 \mathrm{y}$ subescala inteligencia interpersonal 0.58 . La alta correlación encontrada en algunos ítems con el total de la escala era esperable dada la homogeneidad de cada una de las subescalas. Finalmente, los índices de fiabilidad (alfa de Cronbach) para los ocho factores oscilaron entre 0.77 (inteligencia naturalista) y 0.92 (inteligencia musical).

Para la construcción del IAMI-M abreviado (IAMI-M40), se eliminaron los ítems que saturaban en más de un factor y aquellos que saturaban en un factor distinto al original, exceptuando el ítem 2 por su peso en el dominio inteligencia lingüística. Posteriormente, se realizó un estudio de contenido para garantizar la representatividad de cada ítem en el dominio de cada variable. Por último, llevó a cabo un análisis de los ítems para asegurar que cada uno de ellos es esencial y de ninguna manera la consistencia interna del instrumento mejoraría si se eliminaran. En algún caso, se han suprimido los ítems que, a pesar de contar con una carga factorial alta, eran redundantes en su contenido (ítems 3, 8, 29, 48 y 64). La carga factorial de todos los ítems oscilaba entre 0.4 y 0.91 , exceptuando el ítem 50 (0.37). La versión final abreviada (IAMI-M40) quedó compuesta por un total de 40 ítems (véase Apéndice).
Con el fin de analizar la consistencia interna del IAMI-M40, se calcularon los coeficientes alfa de Cronbach (Tabla 2). La selección de ítems para el IAMI-M40 muestra en cada una de las subescalas unos índices de consistencia interna ligeramente inferiores con relación al IAMI-M, pero similares y en algunos casos superiores a los obtenidos en el IAMI-R.

TABLA 2

Comparación de la consistencia interna de las distintas versiones del IAMI

\begin{tabular}{ccccc}
\hline Subescalas & IAMI & IAMI-R & IAMI-M & IAMI-M40 \\
\hline Lingüistica & $0.85(9)$ & $0.76(5)$ & $0.84(9)$ & $0.81(5)$ \\
Lógico-matemática & $0.89(9)$ & $0.89(5)$ & $0.89(9)$ & $0.87(5)$ \\
& & & & \\
Visual-Espacial & $0.91(8)$ & $0.87(5)$ & $0.89(8)$ & $0.85(5)$ \\
Musical & $0.93(8)$ & $0.92(5)$ & $0.93(8)$ & $0.92(5)$ \\
Interpersonal & $0.85(9)$ & $0.79(5)$ & $0.85(9)$ & $0.8(5)$ \\
Cinestésico- & $0.93(9)$ & $0.86(5)$ & $0.92(9)$ & $0.89(5)$ \\
corporal & $0.91(8)$ & $0.91(5)$ & $0.82(8)$ & $0.77(5)$ \\
$\begin{array}{c}\text { Naturalista } \\
\text { Intrapersonal }\end{array}$ & $0.86(9)$ & $0.79(5)$ & $0.87(9)$ & $0.82(5)$ \\
\hline
\end{tabular}

Nota. El número de items aparece entre paréntesis. Fuente: elaboración propia.

Se realizaron análisis descriptivos del IAMI-40 (Tabla 3) y se hallaron las correlaciones entre subescalas. Los índices de correlación oscilaron entre 0.14 y 0.54 , exceptuando los obtenidos entre inteligencia cinestésico-corporal e inteligencias lingüística y musical, que no fueron significativos. La subescala inteligencia cinestésico-corporal mostró una correlación promedio menor que el resto de subescalas. 


\section{TABLA 3}

Tabla de descriptivos y de correlaciones entre las subescalas del IAMI-M40 ( $N=313)$

\begin{tabular}{ccccccccc}
\hline Subescala & Lin & Lom & Epv & Mus & Inter & Cin & Nat & Intra \\
\hline Lin & $5.5(1.6)$ & & & & & & & \\
Lom & $0.34^{* *}$ & $5.45(2.03)$ & & & & & & \\
Epv & $0.2 *^{* *}$ & $0.32^{*}$ & $5.81(1.97)$ & & & & & \\
Mus & $0.33^{* *}$ & $0.21^{* *}$ & $0.24^{* *}$ & $4.86(2.56)$ & & & & \\
Inter & $0.54^{* *}$ & $0.22^{* *}$ & $0.17^{* *}$ & $0.31^{*}$ & $6.78(1.5)$ & & & \\
Cin & 0.07 & $0.22^{*}$ & $0.23^{*}$ & 0.02 & $0.14^{*}$ & $6.88(1.87)$ & & \\
Nat & $0.42^{* *}$ & $0.43^{* *}$ & $0.36^{* *}$ & $0.36^{* *}$ & $0.34^{* *}$ & $0.25^{* *}$ & $5.2(1.68)$ & \\
Intra & $0.46^{* *}$ & $0.27^{* *}$ & $0.2^{*}$ & $0.23^{* *}$ & $0.57^{* *}$ & $0.21^{* *}$ & $0.4^{* *}$ & $7.1(1.48)$ \\
\hline
\end{tabular}

Nota. $*=\mathrm{p}<0.05 ; * *=\mathrm{p}<0.01 ;$ Lin: Inteligencia lingüística; Lom: Inteligencia lógico-matemática; Epv: Inteligencia espacial-visual; Mus: Inteligencia musical; Inter: Inteligencia interpersonal; Cin: Inteligencia cinestésico-corporal; Nat: Inteligencia naturalista; Intra: Inteligencia intrapersonal. En la diagonal se presenta la media y desviación estándar típica de cada una de las subescalas. Fuente: elaboración propia.

\section{Evidencias de validez convergente}

En primer lugar, se presentan los análisis de validez convergente del IAMI-M40 mediante el análisis de correlación entre las puntuaciones de cada una de las subescalas del IAMI-M40 con indicadores de capacidad cognitiva (Gf y Gc) e IE rasgo (Tabla 4). La capacidad cognitiva (Gf) evaluada mediante el PMA-R se relacionó de manera positiva y estadísticamente significativa con las subescalas del IAMIM40: inteligencia lingüística, inteligencia lógicomatemática, inteligencia interpersonal y con inteligencia musical. La capacidad cognitiva (Gc) evaluada mediante el 16PF-r se relacionó de manera positiva y estadísticamente significativa con las subescalas del IAMI-M40: inteligencia lingüística, inteligencia lógico-matemática y con inteligencia interpersonal. La IE rasgo evaluada mediante el TEIQue-ASF se relacionó de forma positiva y estadísticamente significativa con todas las subescalas del IAMI-M40, destacando una mayor correlación con la escala de inteligencia intrapersonal. Por último, se realizó un análisis de las correlaciones medias: en relación con Gf: $r=0.12$; en relación con Gc: $r$ $=0.11$ y en relación con la IE rasgo: $r=0.22$.
TABLA 4

Correlaciones entre las diferentes subescalas del IAMI-40 y otras medidas de inteligencia

\begin{tabular}{lccc}
\hline \multicolumn{1}{c}{ Subescalas } & $\begin{array}{c}\text { Gf } \\
(n=270)\end{array}$ & $\begin{array}{c}\text { Gc } \\
(n=281)\end{array}$ & $\begin{array}{c}\text { IE } \\
(n=291)\end{array}$ \\
\hline Lingüistica & $0.18^{* *}$ & $0.28^{* *}$ & $0.23^{* *}$ \\
Lógico-matemática & $0.24^{* *}$ & $0.15^{*}$ & $0.26^{* *}$ \\
Espacial-Visual & 0.09 & 0.09 & $0.19^{* *}$ \\
Musical & $0.14^{*}$ & 0.05 & $0.14^{*}$ \\
Interpersonal & $0.21^{* *}$ & $0.23^{* *}$ & $0.21^{* *}$ \\
Cinestésico-corporal & -0.04 & -0.04 & $0.2^{* *}$ \\
Naturalista & 0.05 & 0.01 & $0.22^{* *}$ \\
Intrapersonal & 0.07 & 0.09 & $0.33^{* *}$ \\
\hline
\end{tabular}

Nota. $*=p<0.05 ; * *<<0.01 ;$ Gf

(inteligencia fluida); Gc (inteligencia cristalizada); IE (inteligencia emocional rasgo). Fuente: elaboración propia.

Para estudiar específicamente la validez convergente de los subconjuntos "inteligencia académica" e "inteligencias personales" se realizó una serie de análisis de regresión múltiple. Para ello, se aseguró la ausencia de problemas de multicolinealidad (valores de tolerancia $<0.20$; FIV $>4.00$ ). Se introdujeron como variables predictoras las subescalas de las denominadas inteligencias académicas según Gardner (1999), esto es, lingüística, lógico-matemática y espacialvisual, para explorar su poder predictivo sobre los indicadores de inteligencia general (Gf y Gc). Por último, se introdujeron como variables predictoras las subescalas de las denominadas inteligencias personales según Gardner (1999), esto es, interpersonal e intrapersonal, para explorar su poder predictivo sobre el indicador de IE (Tabla 5). 


\section{TABLA 5}

Regresión múltiple de las subescalas del IAMI-40 y Gf, Gc y IE

\begin{tabular}{|c|c|c|c|c|c|c|}
\hline \multirow{3}{*}{$\begin{array}{l}\text { Subescala } \\
\text { LAMI-M40 }\end{array}$} & \multicolumn{2}{|c|}{ Gf } & \multicolumn{2}{|c|}{$\mathrm{Gc}$} & \multicolumn{2}{|c|}{ IE } \\
\hline & \multicolumn{2}{|c|}{$F_{(3.260)}=6.712^{* * * *}, R^{2}{ }^{2}=0.1$} & \multicolumn{2}{|c|}{$F_{(3,277)}=8.168 * * *, \mathrm{R}^{2}{ }_{\mathrm{adj}}=0.07$} & \multicolumn{2}{|c|}{$\begin{array}{c}F_{(2.292)}=18.354^{* * *}, \\
\mathrm{R}_{\mathrm{adi}}^{2}=0.11\end{array}$} \\
\hline & Beta & $\mathrm{I}$ & Beta & $t$ & Beta & $\mathrm{I}$ \\
\hline Lin & 0.115 & 1.796 & 0.259 & $4.169 * * *$ & & \\
\hline Lom & 0.21 & $3.173^{* * *}$ & 0.054 & 0.837 & & \\
\hline Epv & -0.019 & -0.3 & 0.008 & 0.134 & & \\
\hline Inter & & & & & 0.02 & 0.245 \\
\hline Intra & & & & & 0.32 & $4.785 * * *$ \\
\hline \multicolumn{7}{|c|}{$\begin{array}{c}\text { Nota. }{ }^{* * *}=p<0.001 \text {; Lin: inteligencia lingüistica } \\
\text { Lom: Inteligencia lógico-matemática; Epv: } \\
\text { Inteligencia espacio-visual; Inter: Inteligencia } \\
\text { interpersonal; Intra: Inteligencia intrapersonal; } \\
\text { Gf (inteligencia fluida); Gc (inteligencia } \\
\text { cristalizada); IE (inteligencia emocional rasgo) } \\
\text { Fuente: elaboración propia. }\end{array}$} \\
\hline
\end{tabular}

Los resultados obtenidos revelan que la subescala que mejor explica la Gf es la inteligencia lógico-matemática; la subescala que mejor explica Gc es la inteligencia lingüística; y la subescala que mejor explica IE es la inteligencia intrapersonal.

\section{Discusión}

El presente trabajo proporciona evidencias de validez y consistencia interna del IAMI-M40 en una muestra de adolescentes españoles. Los resultados presentan adecuadas evidencias de validez convergente del IAMI-M40 con respecto a medidas de capacidad cognitiva e inteligencia emocional en la dirección esperada. Una apreciación global de los resultados del AFE permite concluir que la gran mayoría de los ítems cargan adecuadamente en los ocho factores, de acuerdo con la teoría de las inteligencias múltiples de Gardner (1999). En este sentido, los resultados del presente estudio replicaron la estructura factorial del IAMI encontrada utilizando muestras no españolas (Pérez et al., 2003; Pérez \& Cupani, 2008), lo que sugiere que la estructura factorial del IAMI-M40 pueda ser equivalente en distintas poblaciones hispanohablantes, algo aún pendiente de comprobación en el futuro. Estos resultados favorables acerca de la validez estructural del IAMI-M40, confieren a este instrumento cierta superioridad psicométrica frente a otros autoinformes que se presentan como evaluación de las IIMM. Por ejemplo, la estructura de ocho factores de inteligencias múltiples no fue encontrada en el caso del autoinforme MIT (Haselbauer, 2005), analizado por Furnham (2009) en una muestra de estudiantes universitarios. Furnham (2009) observó una estructura factorial del MIT compuesta por únicamente tres factores, lo cual es incongruente con los planteamientos teóricos de Gardner (1999). En cambio, en el caso del IAMI-M40, su estructura ha sido derivada de un AFE congruente con el modelo teórico.

El análisis de consistencia interna mostró índices apropiados de fiabilidad (alfa de Cronbach promedio de 0.84 , en un intervalo de 0.77 a 0.92), cumpliendo los estándares deseables para este tipo de instrumentos (p. ej., Nunnally \& Bernstein, 1994), e incluso superando a los hallados en estudios previos con el instrumento original IAMI (Pérez et al., 2003; Pérez \& Cupani, 2008), o en estudios con otros autoinformes homólogos (p. ej., Furnham, 2009; Hernández-Torrano et al., 2014; Llor et al., 2012; Mcmahon et al., 2004).

Un resultado a destacar es que las ocho subescalas correlacionan significativamente entre sí, lo que refuerza la idea de que las ocho inteligencias no son tan independientes entre sí como sugiere la teoría, una cuestión que ya ha sido advertida por autores críticos con la teoría de Gardner (p. ej., Almeida et al., 2010; Reeve \& Bonaccio, 2011; Visser et al., 2006).

Por otra parte, se han hallado evidencias de validez convergente del IAMI-M40 con respecto a medidas de capacidad cognitiva (tanto inteligencia fluida como cristalizada) e IE rasgo. Así, la inteligencia fluida (Gf) se asoció con dos componentes del subgrupo de inteligencias académicas (lingüística, lógicomatemática) y con inteligencias musical e interpersonal. La inteligencia cristalizada (Gc) se relacionó con las subescalas lingüística, lógicomatemática e interpersonal. Cabe destacar que, en línea con lo que sería teóricamente esperable, la inteligencia lógico-matemática mostró mayor correlación con inteligencia fluida, operacionalizada como razonamiento 
inductivo mediante el PMA-R, que con inteligencia cristalizada, operacionalizada como razonamiento verbal mediante el 16-PFR. Asimismo, también de acuerdo con lo teóricamente predecible, la inteligencia lingüística mostró mayor correlación con inteligencia cristalizada que con inteligencia fluida.

Las inteligencias personales evaluadas mediante el IAMI-M40 mostraron validez convergente con la medida de evaluación de la IE rasgo. También es interesante observar que la IE rasgo mostró mayor correlación con inteligencia intrapersonal que con inteligencia interpersonal, lo cual concuerda con la mayor carga del componente intrapersonal de la IE que se puede apreciar en el contenido de los ítems del TEIQue (Brasseur, Grégoire, Bourdu, \& Mikolajczak, 2013). Si bien estos resultados eran esperables dado que las inteligencias intrapersonal e interpersonal constituyen la base del concepto de inteligencia emocional (Salovey \& Mayer, 1990), también sugieren que esta correlación positiva confirmada entre inteligencias personales e IE rasgo podría interpretarse como una evidencia a favor de la conceptualización propuesta por Petrides (2011) de las inteligencias múltiples como rasgos de personalidad y no como tipos de inteligencia. No obstante, es pertinente resaltar que la correlación hallada entre inteligencias personales e IE rasgo puede estar parcialmente inflada como efecto del método de medida compartido, pues para ambos constructos el tipo de instrumento de evaluación utilizado ha sido un autoinforme.

En el trabajo de Furnham (2009), las inteligencias lógico-matemática y lingüística del MIT (Haselbauer, 2005) mostraron validez convergente con un test de conocimiento general, a través de una correlación de $r$ $=0.23, p<0.01$. Hernández-Torrano et al. (2014) encontraron una correlación positiva y significativa entre la escala verbal del Test de Aptitudes Diferenciales (DAT-5) y las inteligencias académicas $(r=0.13, p<0.05)$ evaluadas mediante el MII (Llor et al., 2012), mientras que no hallaron relación significativa entre éstas y la escala de razonamiento abstracto
(Gf) del DAT-5. Las correlaciones entre las escalas del IAMI-M40 y los indicadores objetivos de inteligencia fluida y cristalizada han sido superiores a los de estos estudios previos. Asimismo, las autopercepciones de eficacia evaluadas por el IAMI-40 reflejan cierto grado de capacidad real.

Además, los resultados de los análisis de regresión evidencian validez convergente del IAMI-M40 con respecto a su relación positiva con inteligencia fluida, inteligencia cristalizada e IE, en coherencia con la teoría de Gardner (1999). Específicamente, los análisis de regresión evidencian esta validez convergente para las subescalas de inteligencia lógico-matemática, inteligencia lingüística, e inteligencia intrapersonal, posiblemente las tres clases de inteligencia más importantes en la vida (Murray, 2008).

El IAMI-M40 ha mostrado adecuadas propiedades psicométricas, por lo que con este trabajo se contribuye a hacer disponible, de forma gratuita, una escala válida, fiable, y breve para evaluar las IIMM en adolescentes españoles. Nuestro trabajo aporta un nuevo instrumento de evaluación breve, de fácil administración y accesible, para evaluar las IIMM en el contexto español, lo que implica algunas ventajas para la investigación y la aplicación en los centros educativos. Como señalan Gosling, Rentfrow y Swann (2003), los instrumentos breves pueden facilitar la cooperación de ciertas poblaciones, como la adolescente, que responden mejor ante éstos que ante instrumentos extensos y tediosos.

A pesar de estos hallazgos, nuestra investigación presenta una serie de limitaciones que es necesario subrayar. La primera de las limitaciones hace referencia al carácter no aleatorio e incidental de la muestra empleada, que limita su representatividad y, por tanto, debemos ser precavidos a la hora de generalizar estos resultados. Por otro lado, en futuros estudios sería pertinente realizar análisis factoriales confirmatorios para comprobar si la estructura factorial del IAMI-M40 es replicable y presenta unos índices de ajuste adecuados.

La utilización del IAMI-M40 puede aportar información útil para conocer las características 
y necesidades de cada alumno, lo cual podría ser relevante para diseñar e implementar, tanto programas de intervención dirigidos a mejorar la educación integral, como programas de orientación y acción tutorial dirigidos a la mejora de los procesos de autoconocimiento y toma de decisiones académico-vocacionales del alumnado.

\section{Referencias}

Almeida, L. S., Prieto, M. P., Ferreira, A. I., Bermejo, M. R., Ferrando, M., \& Ferrándiz, C. (2010). Intelligence assessment: Gardner multiple intelligence theory as an alternative. Learning and Individual Differences, 20, 225-230. doi:10.1016/j.lindif.2009.12.010

Armstrong, T. (1999). Inteligencias múltiples en el aula. Buenos Aires: Manantial.

Bandura, A. (2006). Guide for creating selfefficacy scales. En F. Pajares \& T. Urdan (Eds.), Self-efficacy beliefs in adolescents (pp. 307-337). Greenwich, CT: Information Age Publishing.

Beavers, A. S., Lounsbury, J. W., Richards, J. K., Huck, S. W., Skolits, G. J., \& Esquivel, S. L. (2013). Practical considerations for using exploratory factor analysis in educational research. Practical Assessment, Research and Evaluation, 18(6). Recuperado de http://pa reonline.net/pdf/v18n6.pdf

Brasseur, S., Grégoire, J., Bourdu, R., \& Mikolajczak, M. (2013). The Profile of Emotional Competence (PEC): Development and validation of a selfreported measure that fits dimensions of emotional competence theory. PLoS One, 8(5), e62635. http://dx.doi.org/10.1371/ journal.pone.0062635

Brody, N. (1992). Intelligence (2nd. ed.). New York: Academic Press.

Carroll, J. B. (1993). Human cognitive abilities: A survey of factor-analytic studies. Cambridge, UK: Cambridge University Press.

Cattell, R. B., \& Cattell, A. K., \& Cattell, H. E. P. (1993). Sixteen Personality Factor
Questionnaire (5th. ed.). Champaign, Il: Institute for Personality and Ability Testing, Inc.

Chan, D. W. (2001).Assessing giftedness of Chinese secondary students in Hong Kong: A multiple intelligences perspective. High Ability Studies, 12, 215-234. http:// dx.doi.org/10.1080/13598130120084348

Cronbach, L. J. (1949). Essentials of psychological testing (2a. ed.). Nueva York: Harper \& Row.

Ferrando, M., Prieto, M. D., Almeida, L. S., Ferrándiz, C., Bermejo, R., LópezPina, J. A.,... Fernandez, M. C. (2011). Trait emotional intelligence and academic performance: Controlling for the effects of IQ, personality, and self-concept. Journal of Psychoeducational Assessment, 29(2), 150-159. http:// dx.doi.org/10.1177/0734282910374707

Furnham, A. (2009). The validity of a new, self-report measure of multiple intelligence. Current Psychology, 28, 225-239. http:// dx.doi.org/10.1007/s12144-009-9064-z

Gardner, H. (1983). Frames of mind: The theory of multiple intelligences. Nueva York: Basic Books.

Gardner, H. (1998). A reply to Perry D. Klein's "Multiplying the problems of intelligence by eight". Canadian Journal of Education, 23(1), 96-102. http://dx.doi.org/10.1016/ j.intell.2006.04.002

Gardner, H. (1999). Intelligence reframed. Multiple intelligences for the 21st. Century. Nueva York: Basic Books.

Gardner, H. (2006). On failing to grasp the core of MI theory: A response to Visser et al. Intelligence, 34, 503-505. http:// dx.doi.org/10.1016/j.intell.2006.04.002

Gosling, S. D., Rentfrow, P. J., \& Swann, W. B. (2003). A very brief measure of the Big-Five personality domains. Journal of Research in Personality, 37, 504-528. http://dx.doi.org/10.1016/ S0092-6566(03)00046-1

Haselbauer, N. (2005). What's your IQ?. Nueva York: Barnes \& Noble Books. 
Hendrickson, A. E., \& White, P. O. (1964). Promax: A quick method for rotation to oblique simple structure. The British Journal of Statistical Psychology, 17(1), 65-70. http://dx.doi.org/10.1111/ j.2044-8317.1964.tb00244.x

Hernández-Torrano, D., Ferrándiz, C., Ferrando, M., Prieto, L., \& Fernández, C. (2014). The theory of multiples intelligences in the identification of high-ability students. Anales de Psicología, 30(1), 192-200. http:// dx.doi.org/10.6018/analesps.30.1.148271

Hertzog, C., \& Bleckley, M. K. (2001). Age differences in the structure of intelligence: Influences of information processing speed. Intelligence, 29, 191-217. http://dx.doi.org/10.1016/ S0160-2896(00)00050-7

Klein, P. D. (1997). Multiplying the problems of intelligence by eight: A Critique of Gardner's Theory. Canadian Journal of Education, 22(4), 377-394. http:// dx.doi.org/10.2307/1585790

Klein, P. D. (1998). A response to Howard Gardner: Falsifiability, empirical evidence, and pedagogical usefulness in educational psychology. Canadian Journal of Education, 23(1), 103-112. http:// dx.doi.org/10.2307/1585969

Kline, P. (1994). An easy guide to factor analysis. Londres: Routledge.

Llor, L., Ferrando, M., Ferrándiz, C., Hernández, D., Sáinz, M., Prieto, M. D., \& Fernández, M. D. (2012). Inteligencias múltiples y alta habilidad. Aula Abierta, 40(1), 27-38.

McMahon, S. D., Rose, D. S., \& Parks, M. (2004). Multiple intelligences and reading achievement: An examination of the Teele Inventory of multiple intelligences. Journal of Experimental Education, 73(1). 41-52. http://dx.doi.org/10.3200/jexe.71.1

Montero, I., \& León, O. G. (2007). A guide for naming research studies in Psychology. International Journal of Clinical and Health Psychology, 7(3), 847-862.

Muñiz, J., Elosua, P., \& Hambleton, R. K. (2013). Directrices para la traducción y adaptación de los test. Psicothema,
25(2), 151-157. http://dx.doi.org/10.7334/ psicothema2013.24

Murray, C. (2008). Real education. Nueva York: Crown Forum.

Nunnally, J. C., \& Bernstein, I. H. (1994). Psychometric theory (3rd. ed.). Nueva York: McGraw-Hill.

O'Connor, R. M., \& Little, I. S. (2003). Revisiting the predictive validity of emotional intelligence: Selfreport versus ability-based measures. Personality and Individual Differences, 35, 1893-1902. http://dx.doi.org/10.1016/ S0191-8869(03)00038-2

Pérez, E., Beltramino, C., \& Cupani, M. (2003). Inventario de Autoeficacia para Inteligencias Múltiples. Fundamentos teóricos y estudios psicométricos. Evaluar, 3, 36-60.

Pérez, E., \& Cupani, M. (2008). Inventario de Autoeficacia para Inteligencias Múltiples Revisado (IAMI-R). Revista Latinoamericana de Psicología, 40(1), 47-58.

Pérez, L., \& Beltrán, J. (2006). Dos décadas de "inteligencias múltiples": implicaciones para la psicología de la educación. Papeles del Psicólogo, 27(3), 147-164.

Pérez-González, J.C., \& Sánchez-Ruiz, Maㅗ J. (2014). Trait emotional intelligence anchored within the big five, big two and big one frameworks. Personality and Individual Differences, 65, 53-58.

Petrides, K. V. (2011). Ability and trait emotional intelligence. En T. Chamorro-Premuzic, A. Furnham \& S. von Stumm (Eds.), The Blackwell-Wiley handbook of individual differences (pp. 656-678). Nueva York: Wiley.

Petrides, K.V., Sangareau, Y., Furnham, A., \& Frederickson, N. (2006). Trait emotional intelligence and children's peer relations at school. Social Development, 15(3), 537-547. http://dx.doi.org/10.1111/ j.1467-9507.2006.00355.x

Pizarro, R., \& Clark, S. (2000). Inteligencia múltiple lógico-matemática y aprendizajes escolares científicos. Revista de Psicología, 9(1), 75-89. 
Pizarro, R., Colarte, P., Machuca, L., Donoso, F., Martínez, M., \& Walter, I. (2002). Análisis psicométrico de las Escalas de Inteligencias Múltiples MIDAS-kids. Revista de Psicología de Chile, 11(2), 111-124.

Reeve, C. L., \& Bonaccio, S. (2011). The nature and structure of "intelligence." En T. Chamorro-Premuzic, A. Furnham \& S. von Stumm (Eds.), Handbook of individual differences (pp. 187-216). Oxford, England: Wiley-Blackwell.

Roberts, R. D., \& Lipnevich, A. A. (2011). From general intelligence to multiple intelligences: Meanings, models, and measures. En K. R. Harris, S. Graham \& T. Urdan (Eds.), APA educational psychology handbook (Vol. 2, pp. 33-57). Washington, DC: American Psychological Association.

Román, F. J., Abad, F. J., Escorial, S., Burgaleta, M., Martínez, K., Álvarez-Linera, J.,... Colom, R. (2014). Reversed hierarchy in the brain for general and specific cognitive abilities: A morphometric analysis. Human Brain Mappin, 35, 3805-3818. http:// dx.doi.org/10.1002/hbm.22438

Salovey, P., \& Mayer, J. D. (1990). Emotional intelligence. Imagination, Cognition and Personality, 9(3), 185-211. http://dx.doi.org/10.2190/ DUGG-P24E52WK-6CDG

Sánchez-Ruiz, M. J., Hernández-Torrano, D., Pérez-González, J. C., Batey, M., \& Petrides, K. V. (2011). The relationship between trait emotional intelligence and creativity across subject domains. Motivation and Emotion, 35, 461-473. http://dx.doi.org/10.1007/ s11031-011-9227-8

Shearer, C. B. (1999). The MIDAS challenge! A guide to career success. Kent, $\mathrm{OH}$ : MI Research and Consulting.

Shearer, C. B. (2004). Using a multiple intelligences assessment to promote teacher development and student achievement. Teachers College Record, 106(1), 147-162. http:// dx.doi.org/10.1111/ j.1467-9620.2004.00325.x
Teele, S. (1992). Teele Inventory of Multiple Intelligences. Redlands, CA: Sue Teele and Associates.

Thurstone, L. L., \& Thurstone, T. G. (1941). Factorial studies of intelligence. Chicago: University of Chicago Press.

Visser, B. A., Ashton, M. C., \& Vernon, P. A. (2006). g and the measurement of Multiple Intelligences: A response to Gardner. Intelligence, 34, 507-510. http:// dx.doi.org/10.1016/j.intell.2006.02.004

Waterhouse, L. (2006). Multiple intelligences, the Mozart effect, and emotional intelligence: A critical review. Educational Psychologist, 41, 207-225. http:// dx.doi.org/10.1207/s15326985ep4104_1

\section{ANEXO A}

\section{Inventario de autoeficacia para inteligencias múltiples para adolescentes (iami-m40).}

\section{Instrucciones}

Este inventario comprende 40 ítems y cada uno de ellos describe una actividad específica. Se solicita que evalúes cuánta confianza posees en poder realizar exitosamente cada actividad utilizando una escala que va desde 1 (No puedo desempeñar esta actividad) a 10 (Estoy completamente seguro de poder desempeñar esta actividad exitosamente). 


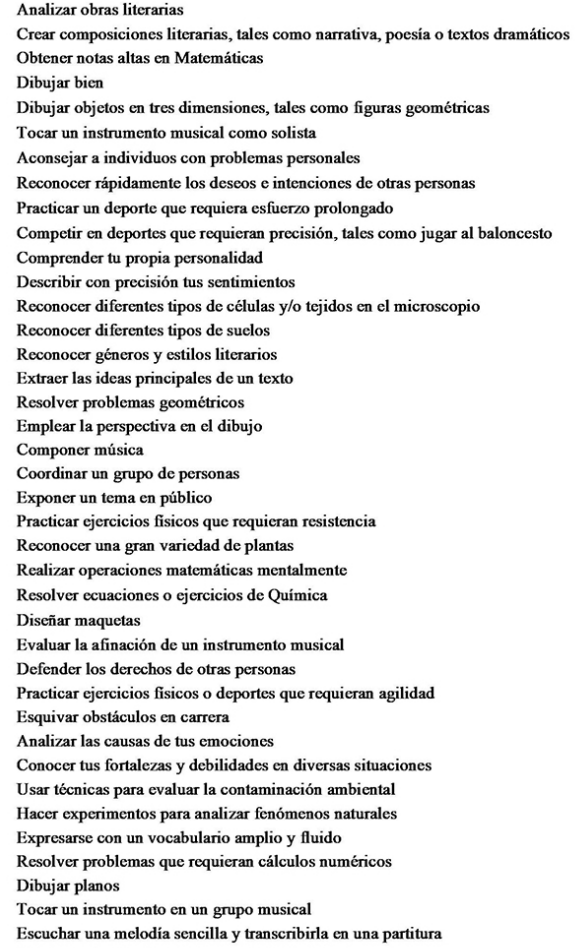

Nota: Para el cálculo de la puntuación se deben sumar las valoraciones puestas a cada uno de los ítems que componen cada subescala.

Posteriormente, se divide esa suma por el número de ítems y el resultado se puede interpretar en el intervalo de 1 a 10, coincidiendo con la escala de respuesta del cuestionario. Los ítems que corresponden a cada una de las subescalas son los siguientes: I. Lingüística $=(1,2,15,16,35) / 5$ II. Lógico-matemática $=(3,17,24,25,36) / 5$ III. Espacial-visual $=(4,5,18,26,37) / 5 \mathrm{IV}$. Musical $=$ $(6,19,27,38,39) / 5 \mathrm{~V}$. Interpersonal $=(7,8,20$, $21,28) / 5$ VI. Cinestésico-corporal $=(9,10,22$, $29,30) / 5$ VII. Intrapersonal $=(11,12,31,32$, 40) $/ 5$ VIII. Naturalista $=(13,14,23,33,34) / 5$

\section{Notas}

* Artículo de investigación. 